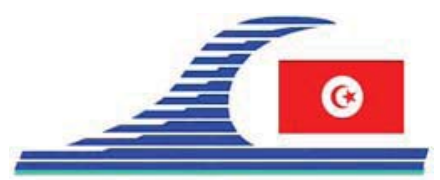

Conférence Méditerranéenne Côtière et Maritime

EDITION 1, HAMMAMET, TUNISIE (2009)

Coastal and Maritime Mediterranean Conference

Disponible en ligne - http://www.paralia.fr-Available online

\title{
Prédiction de l'affaissement des sols côtiers par des essais ultrasoniques
}

\author{
Med Salah LAOUAR ${ }^{1}$, Khelifa ABBECHE ${ }^{2}$, Farid MESSAOUD ${ }^{1}$
}

1. Département de Génie Civil Université de Tébessa, Algérie.

2. Département de Génie Civil Université de Batna, Algérie.

c_laouar@yahoo.fr

\section{Résumé :}

Des transformations importantes dans les sols côtiers surtout en régions subtropicales, arides et semi-arides sont dues essentiellement aux effets indirects liés aux flots de boue sédimentaires mous, provoqués par les changements climatiques et la montée du niveau des mers. Des phénomènes tels que : l'ensablement, l'érosion et l'envasement modifient les propriétés initiales de ces sols donnant la naissance à des structures macro poreuses ouvertes qui possèdent une grande quantité de particules limoneuses. Face aux effets d'aridité et de réhumidification où leur comportement devient similaire aux sols effondrables, ce type de sol est instable comme support de fondation. L'objectif de cette recherche est de prédire le potentiel d'effondrement de ces sols après inondations par des essais expérimentaux. Les résultats ont montré que l'approche expérimentale utilisée dans cette recherche peut être utilisée avec succès pour comprendre le problème d'affaissement des sols.

\section{Mots-clés :}

Effondrement - Ultrasons - Sols côtiers - Sols évolutifs

\section{Introduction}

Les risques croissants d'inondation sur la côte résultent des tendances climatiques moins prévisibles et de l'élévation du niveau de la mer. Malgré la réalisation de divers ouvrages de protection et de défense le long des littoraux, le sol côtier reste sensible aux risques liés aux effets de la houle, de l'érosion et de transfert hydro sédimentaire (HOSKING \& MOORE, 2001). L'interaction vagues-plage combinée avec les changements climatiques modifie l'état initial de ces sols donnant des structures évolutives susceptibles d'effondrement et de glissement. Parmi les dégâts enregistrés on note les inondations qu'a connues Alger en 2001 et qui a fait environ 1000 morts. Au cours de ces dernières décennies de nombreuses études scientifiques ont été réalisées visant à comprendre les incertitudes impliquées dans le phénomène d'effondrement des sols côtiers. La littérature révèle que la majorité des recherches a été consacrée aux mécanismes d'affaissement et aux méthodes d'identification et de traitement. 
Malgré l'existence d'une grande gamme d'équipements ultrasoniques et la vaste utilisation de ce procédé dans divers domaines, peu d'attention a été accordée à cette technique dans la géotechnique maritime. Ce travail présente en plus des résultats d'essais de compressibilités, des courbes expérimentales originales des essais non destructifs aux ultrasons.

\section{Caractéristiques des matériaux}

Les essais ont été effectués sur six sols reconstitués composés de sables et de kaolin en différentes proportions, pour lesquels l'application des critères d'affaissement, rapportés par AYADAT et BELLILI (1995), montre que ceux-ci sont évolutifs.

Deux types de sables tamisés à $2 \mathrm{~mm}$ sont utilisés pour la reconstitution des sols; sable de dunes et sable de oued lavés et séchés, leurs caractéristiques sont:

Sable de dunes : granulométrie comprise entre 0,08 et $2 \mathrm{~mm}$, dont $1,36 \%$ des particules inférieures à $80 \mu \mathrm{m}$, coefficient d'uniformité de 3,93, coefficient de courbure de 1,31, équivalent de sable de 75,16\%, et densité spécifique des grains $\mathrm{Gs}=2,64$.

Sable d'oued: granulométrie comprise entre 0,08 et $2 \mathrm{~mm}$, dont $3,01 \%$ des particules inférieures à $80 \mu \mathrm{m}$, coefficient d'uniformité de 2,18, coefficient de courbure de 0,94, équivalent de sable de $68,69 \%$, et $\mathrm{Gs}=2,66$.

Le kaolin : est extrait de la mine d'Elmilia (Algérie) de diamètre inférieure à $80 \mu \mathrm{m}$, de couleur jaunâtre, utilisé généralement dans la fabrication de la porcelaine et les produits céramiques. Ses caractéristiques sont: $\mathrm{Gs}=2,4$, limite de liquidité de 65,83\%, limite de plasticité de $39,64 \%$, pourcentage inférieure à $2 \mu \mathrm{m}$ égal à $43 \%$,

Les sols S1, S2 et S3 sont reconstitués avec des sables de dunes et du kaolin, tandis que les sols S4, S5 et S6 sont reconstitués avec des sables de oued et du kaolin. Les caractéristiques géotechniques des sols reconstitués sont présentées dans le tableau 1.

Tableau 1. Caractéristiques des sols reconstitués.

\begin{tabular}{lllllll}
\hline Designation & S1 & S2 & S3 & S4 & S5 & S6 \\
\hline \% kaolin & 15 & 35 & 50 & 20 & 30 & 40 \\
\% sable de dunes & 85 & 65 & 50 & - & - & - \\
\% sable de oued & - & - & - & 80 & 70 & 60 \\
$\mathrm{G}_{\mathrm{S}}$ & 2,65 & 2,59 & 2,46 & 2,62 & 2,56 & 2,48 \\
$\mathrm{~W}_{\mathrm{L}} \%$ & 16,47 & 26,63 & 35,37 & 18,47 & 28,97 & 33,42 \\
$\mathrm{~W}_{\mathrm{P}} \%$ & 11,03 & 15,37 & 20,87 & 11,95 & 14,77 & 19,03 \\
$\gamma_{\mathrm{d} \max }\left(\mathrm{g} / \mathrm{cm}^{3}\right)$ & 2,04 & 1,95 & 1,84 & 1,95 & 1,82 & 1,75 \\
$\mathrm{~W}_{\text {opt }} \%$ & 8,62 & 9,43 & 13,88 & 12,82 & 14,67 & 17,82 \\
$\%<2 \mu \mathrm{m}$ & 4,91 & 11,73 & 16,74 & 7,03 & 9,84 & 14,12 \\
\hline
\end{tabular}

\section{Programme des essais}

Le programme expérimental comporte deux séries d'essais : essais œdométriques, réalisés selon la procédure de JENNINGS et KNIGHT (1975), et essais aux ultrasons 
menés à l'aide d'analyseur ultrasonique sur des éprouvettes de même taille que pour les essais œdométriques, chaque série d'essais comprend 96 tests. Les paramètres retenus dans chaque test sont: les teneurs en eau : $2 \%, 4 \%, 6 \%$ et $8 \%$, et les degrés de compactage : 10, 25,40 et 60 coups. L'énergie de compactage totale Ec mise en jeu pour une éprouvette de sol, étant le travail de la masse $M$ sur une hauteur $h$, elle s'exprime par la relation : $\mathrm{Ec}=\mathrm{M} \mathrm{g}$ h $\mathrm{n}$

où : $\mathrm{n}$ est le nombre de coups de la masse $\mathrm{M}$, et $\mathrm{g}$ l'accélération de la pesanteur.

\section{Résultats}

Les caractéristiques de compressibilité et de consistance dépendent essentiellement de la teneur en eau initiale (fig. 1). Les résultats obtenus montrent clairement l'influence de certains paramètres tels que la teneur en kaolin, la teneur en eau et l'énergie de compactage sur le potentiel d'affaissement et la vitesse ultrasonique. Le potentiel d'effondrement peut être exorbitant, si la teneur en eau initiale est faible. La vitesse ultrasonique et le potentiel d'affaissement d'un sol côtier sont inversement proportionnels. Les figures 2 et 3 représentent les variations des vitesses ultrasoniques en fonction des teneurs en eau et des énergies de compactage.

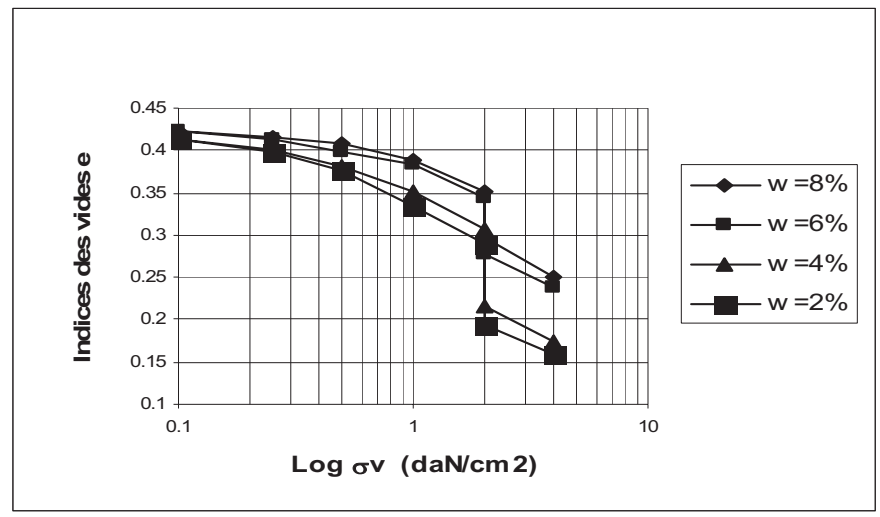

Figure 1. Courbes oedométriques des sols $S 1(E=25$ coups $)$.

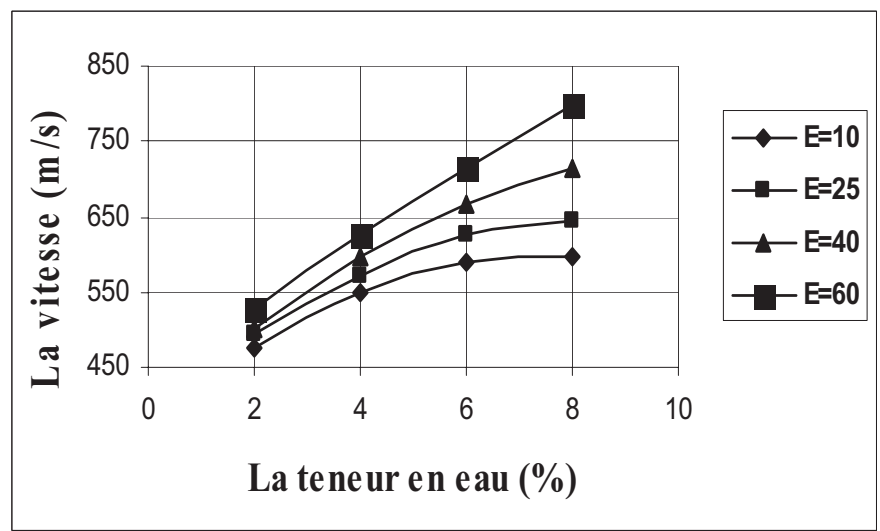

Figure 2. La vitesse ultrasonique en fonction de la teneur en eau (sol 2). 


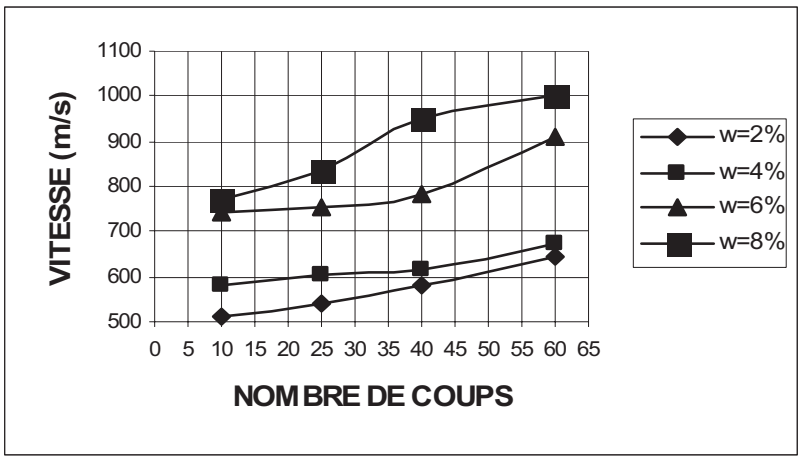

Figure 3. La vitesse ultrasonique en fonction de nombre de coups (sol 5).

\section{Conclusions}

Les principales conclusions qu'on peut tirer de cette étude se résument comme suit :

- Pour une teneur en eau inférieure à l'optimum de Proctor, il existe une énergie de compactage au delà de laquelle l'affaissement ne se produit pas.

- Proposition d'une nouvelle approche expérimentale de prédiction de l'affaissement des sols côtiers : facile et rapide, elle est basée sur des essais ultrasoniques. Cette procédure peut être effectuée au laboratoire ou in situ, sur des échantillons de forme quelconque, intacts ou remaniés. Les résultats obtenus dépendent de la granulométrie, de l'état de compacité du sol et de la teneur en eau. Les vitesses ultrasoniques sont limitées comme suit : $\mathrm{Si} \mathrm{V} \leq 500 \mathrm{~m} / \mathrm{s}$, l'affaissement se manifeste ;

$$
\text { Si V }>1000 \mathrm{~m} / \mathrm{s} \text {, le risque d'affaissement est écarté. }
$$

Entre ces deux limites, l'effondrement peut se produire, il dépend de la teneur en eau et de l'état de compacité.

\section{Références bibliographiques}

AYADAT T, BELLILI F. (1995). Sols susceptibles d'affaissement: Identification mécanique et traitement. Revue Algérie Équipement, $\mathrm{n}^{\circ} 20$, pp 18-23.

HOSKING A. MOORE R. (2001). Preparing for the impacts of climate change. Report for SCOPAC by Halcrow Ltd. Swindon, SCOPAC.

JENNINGS J E., KNIGHT K. (1975). The additional settlement of foundation due to collapse of sandy soils on wetting. Proc. 4th Inter. Conf. on Soil Mechanics and Foundation Engineering, 1, pp 316-319. 